\title{
Pediatric anaphylaxis and hyper IgE syndrome
}

This article was published in the following Dove Press journal:

Journal of Asthma and Allergy

28 February 2017

Number of times this article has been viewed

\section{Amrita Dosanjh \\ Department of Pediatrics, Rady Childrens Hospital, San Diego, CA, USA}

Correspondence: Amrita Dosanjh Email pulmd@aol.com
Abstract: Patients with autosomal-dominant (AD) hyper immunoglobulin E (IgE) syndrome (HIES) or Job syndrome develop frequent dermatologic and pulmonary infections. As patients have an extreme elevation of IgE levels, this database analysis study sought to study the association between AD HIES, Job syndrome, and anaphylaxis. HIES is a heterogeneous group of immune disorders characterized by extremely elevated levels of serum IgE. Although the molecular defects and clinical phenotypes found in association with Job syndrome are well characterized, the association with severe allergic reactions and anaphylaxis is a subject of ongoing investigation. Keywords: anaphylaxis, hyper IgE syndrome, allergy, pediatrics

\section{Introduction}

Job syndrome is one of the hyper immunoglobulin E (IgE) syndrome (HIES) conditions characterized by signal transducer and activator of transcription 3 (STAT3) signaling mutations. Patients with Job syndrome have characteristic clinical features in association with mutations in STAT3 signaling. These include marked elevation in serum IgE and susceptibility to Staphylococcal infections of the pulmonary tract and skin. ${ }^{1}$ One of the hallmark features of the disease includes the formation of abscesses. On the basis of the elevation of serum IgE levels, it was hypothesized that there would be a lower incidence of clinical anaphylaxis among pediatric Job syndrome patients.

\section{Methods}

An electronic medical record database, including both inpatient and outpatient records, was utilized. The database from a large urban children's medical center was searched from January 1, 2009 to December 31, 2014, using the International Classification of Diseases, Ninth Revision (ICD-9) codes assigned to each diagnosis, Job syndrome, HIES, and anaphylaxis. The ICD-9 codes used corresponding to each diagnosis were as follows: asthma, 493.00-493.99; Job syndrome, 288.1; and anaphylactic shock, 995.61 (peanut) and 995.67 (milk).

The anonymous data generated for anaphylactic shock, asthma with anaphylactic shock, and Job syndrome/HIES were used to identify patients. The total number of patients was identified with 1) Job syndrome/HIES; 2) asthma with anaphylactic shock; 3) anaphylactic shock with and without peanut allergy, and with and without milk allergy; and 4) Job syndrome/HIES with anaphylactic shock. The database search was conducted with the medical center health information support and did not involve personal health information analysis. The cohorts with asthma and food allergy 
were compared with the Job syndrome cohort. The cohort identified those patients with HIES and Job syndrome. The comparison groups were asthma and food allergies. Both groups are considered at risk for anaphylaxis.

The study was exempt from IRB review because there was no use of personal health information, as discussed with the University of California IRB office. The only information extracted was based on coding.

\section{Results}

The total number of patients with Job syndrome/HIES identified in the database was 18 . Among the 22,890 patients evaluated with asthma, 29 had experienced anaphylactic shock $(0.1266 \%)$. Of the 614 total anaphylactic patient cases identified, $286(46.5 \%)$ had anaphylaxis with no determined specific cause. There were 250 cases $(40.7 \%)$ of peanut allergen-induced anaphylaxis and 78 cases $(12.7 \%)$ were because of milk products. Some patients may have been coded for one or more causes of shock.

Despite the elevation of IgE associated with HIES/Job syndrome, there were no cases of Job syndrome/HIES and anaphylaxis.

\section{Statistical analysis}

A one- and two-tailed $t$-test showed that there was no statistically significant difference when comparing asthma and anaphylaxis and HIES with anaphylaxis. A $p$-value of $<0.05$ was defined as significant.

\section{Discussion}

This study shows that there were no identified cases of anaphylaxis among the Job syndrome/HIES population studied. This low incidence is in contrast to established higher rates of anaphylaxis among atopic conditions associated with high levels of serum IgE. The incidence of anaphylaxis among children and adolescents has been reported as between $0.05 \%$ and $2 \%{ }^{2}$ This study identified $>600$ cases of anaphylaxis, during the same time period at the same medical center, among the nonHIES pediatric population. The association between allergic disease and autosomal-dominant (AD) HIES was studied in a murine model. HIES is associated with mutations in the STAT family of transcriptional factors. These mutations result in a very elevated serum IgE level, usually without any evidence of atopic disease. The authors found that in a murine model of mast cell degranulation, mast cell-induced anaphylaxis was blunted in the mutant mice. The animals treated with a C188-9 STAT3 inhibitor demonstrated altered physiologic responses. The responses measured included body temperature and survival. Those treated with the inhibitor had longer survival and less change in body temperature. ${ }^{3}$ In a cellular model described in the same citation, human umbilical vein cells from AD HIES patients or control cells treated with a STAT3 inhibitor demonstrated aberrant responses to mast cell mediators, such as histamine or platelet-activating factor. The cells from two newborns with HIES were less permeable than wild-type cells when exposed to mast cell mediators. ${ }^{3}$ These findings suggest that STAT3 mutations confer resistance to mast cell degranulation.

A clinical study of food allergies and adult HIES conducted a clinical evaluation of food allergies in a cohort of 71 patients with AD HIES compared with healthy control subjects $(n=41)$ and atopic subjects $(n=65)$. The results indicated that fewer patients with AD HIES developed food allergies and anaphylaxis than atopic patients with marked serum $\operatorname{IgE}$ elevation, eczema, and no STAT3 mutations. The study reported that fewer patients with AD HIES had anaphylaxis to a food allergen than atopic controls (8.5\% vs. $33.3 \%)$. The AD HIES patients did demonstrate that they had specific IgE to food allergens. When comparing peanut anaphylaxis between the groups, there were no cases of peanut-induced anaphylaxis found in the patients with HIES. ${ }^{4}$ The authors concluded that STAT3 mutations may play a role in protecting the individual from food allergies as a trigger for anaphylaxis. In studying the activation of a key regulatory cell, basophils from HIES patients were less sensitive to IgE cross-linking than basophils from non-allergic subjects. ${ }^{4}$ This study demonstrates that among the pediatric cases identified, there have been no cases of anaphylaxis among the HIES patients. This is in contrast to a large number of children without HIES who did have anaphylaxis due to peanut or milk allergens. This study finds support for a potential protective effect of STAT3 mutations in preventing anaphylaxis and demonstrates this finding in a pediatric population. Further studies are warranted to identify the molecular and cellular pathways involved in reducing vascular permeability during mast cell degranulation among children with HIES.

\section{Acknowledgment}

The author would like to thank the Health Information Service Department.

\section{Disclosure}

The author reports no conflicts of interest in this work.

\section{References}

1. Holland SM, DeLeo FR, Elloumi HZ, Hsu AP, Uzel G, Brodsky N. STAT3 mutations in the hyper-IgE syndrome. $N$ Engl J Med. 2007;357(16): 1608-1619.

2. Lieberman P, Camargo CA Jr, Bohike K, et al. Epidemiology of anaphylaxis: findings of the American College of Allergy, Asthma and Immunology. Ann Allergy Asthma Immunol. 2006;97(5):596-602. 
3. Hox V, O’Connell MP, Lyons JJ, et al. Diminution of signal transducer and activator of transcription 3 signaling inhibits vascular permeability and anaphylaxis. J Allergy Clin Immunol. 2016;138(1):1-13.
4. Siegel A, Stone K, Curuse G, et al. Diminished allergic disease in patients with STAT3 mutations reveals a role for STAT3 signaling in mast cell degranulation. J Allergy Clin Immunol. 2013;132(6):1388-1396.
The Journal of Asthma and Allergy is an international, peer-reviewed open access journal publishing original research, reports, editorials and commentaries on the following topics: Asthma; Pulmonary physiology; Asthma related clinical health; Clinical immunology and the immunological basis of disease; Pharmacological interventions and new therapies. This journal is included in PubMed. The manuscript management system is completely online and includes a very quick and fair peer-review system, which is all easy to use. Visit http://www. dovepress.com/testimonials.php to read real quotes from published authors.

Submit your manuscript here: https://www.dovepress.com/journal-of-asthma-and-allergy-journal 